\title{
Modelling large motion events in fMRI studies of patients with epilepsy
}

\author{
Louis Lemieux $^{1 *}$; Afraim Salek-Haddadi ${ }^{1}$; Torben E. Lund ${ }^{2}$; Helmut Laufs ${ }^{1}$; David Carmichael ${ }^{1}$ \\ ${ }^{1}$ Department of Clinical and Experimental Epilepsy, \\ Institute of Neurology, University College of London \\ Queen Square, London WC1N 3BG, United Kingdom \\ And \\ MRI Unit, National Society for Epilepsy \\ Chesham Lane \\ Chalfont St. Peter, Buckinghamshire SL9 0RJ, United Kingdom \\ ${ }^{2}$ Danish Research Centre for Magnetic Resonance MR Department, Section 340, Copenhagen \\ University Hospital, Hvidovre, Kettegaard Allé 30, DK-2650 Hvidovre, Denmark.
}

* Corresponding author:

Professor Louis Lemieux

MRI Unit, National Society for Epilepsy

Chesham Lane

Chalfont St. Peter, Buckinghamshire SL9 0RJ, United Kingdom

Tel.: +44-1494-601361

Fax: +44-1494-875666. 


\begin{abstract}
EEG-correlated fMRI can provide localisation information on the generators of epileptiform discharges in patients with focal epilepsy. To increase the technique's clinical potential, it is important to consider ways of optimising the yield of each experiment while minimizing the risk of false positive activation. Head motion can lead to severe image degradation and result in false positive activation, and is usually worse in patients than in healthy subjects. We performed General Linear Model (GLM) fMRI data analysis on simultaneous EEG-fMRI data acquired in 34 cases with focal epilepsy. Signal changes associated with large inter-scan motion events (head jerks) were modelled using modified design matrices that include 'scan nulling' regressors. We evaluated the efficacy of this approach by mapping the proportion of the brain for which F-tests across the additional regressors were significant. In $95 \%$ of cases, there was a significant effect of motion in $50 \%$ of the brain or greater; for the scan nulling effect, the proportion was $36 \%$; this effect was predominantly in the neocortex. We conclude that careful consideration of the motionrelated effects in fMRI studies of patients with epilepsy is essential and that the proposed approach can be effective.
\end{abstract}




\section{Introduction}

Noise is a key factor in the ability of functional Magnetic Resonance Imaging (fMRI) to reveal significant correlation between Blood Oxygenation Level Dependant (BOLD) changes and postulated or observed changes in brain activity. For any voxel, high variance in the un-modelled signal (i.e. the residuals) reduces sensitivity to the effects of interest. Therefore the inclusion of nuisance effects such as signal drift, motion-related effects, physiological noise into models of the fMRI signal, in addition to the effects of interest, can increase sensitivity ${ }^{1}$.

The investigation of the hemodynamic correlates of interictal or ictal epileptiform activity using EEG-correlated fMRI has provided important new knowledge on the location of the generators of such activity. The issue of localisation is crucial in the presurgical assessment of patients with drug-resistant epilepsy, with direct implications for treatment. The BOLD maps obtained using this technique commonly reveal patterns that are in broad anatomical agreement with the generator localisation inferred from independently acquired electroclinical data ${ }^{2-4}$. However, depending on how flexibly the data is analysed, the maps may contain additional clusters remote from the presumed generator with time courses that do not match the normal physiological response, rendering interpretation difficult ${ }^{5,6}$. Equally importantly, the technique's yield is limited, with roughly two thirds of selected cases (in whom epileptiform EEG abnormalities are observed during fMRI acquisition) showing significant activation ${ }^{4,7}$. In this work we focus on modelling motionrelated signal changes to address the above limitations.

The problem of head motion-related nuisance effects in fMRI has long been recognised ${ }^{8}$. In general, head motion is detrimental to fMRI in two ways: on one hand, it can give rise to artefacts when correlated with the stimuli or events of interest (false positive activations), and on the other it can lead to reduced sensitivity when not accounted for properly through an increase in residual variance (false negative activations) ${ }^{9}$. Despite this, motion-related effects are not always considered in the modelling of fMRI data, and in particular data acquired in patients with epilepsy. 
The degree of motion that is sufficient to produce the aforementioned detrimental effects is very small, of the order of $1 \mathrm{~mm}$ or less ${ }^{8}$. While head motion can be reduced by mechanical means, such as bite bars and vacuum cushions, it cannot always be eliminated and is limited by considerations of subject comfort and safety.

Numerous post-acquisition strategies for limiting the impact of head motion on the fMRI results have been proposed. The single most important and universally adopted step is retrospective realignment of the image time series using image co-registration ${ }^{10}$. Although spatial realignment reduces inter-scan differences due to variations in the field-of-view's anatomical coverage, and can be adapted to account for differences in the degree of distortion and signal drop-out (which are functions of head position) ${ }^{11}$, it does not generally remove all signal differences associated with motion. Residual motion-induced signal can be modelled as linear or non-linear functions of the scan realignment parameters derived from the procedure ${ }^{12}$. This modelling approach can result in increased sensitivity, and reduced likelihood of motion-related (false) activation, and has been shown to be statistically efficient ${ }^{9}$. However, the latter benefit can also mean that true (i.e. linked to the effects of interest) activation may also be removed in the presence of stimulus-correlated motion.

In the context of IED-correlated fMRI's potential clinical use, an additional consideration is the propensity of patients to move more than healthy subjects. Our experience shows that fMRI time series are often compromised by large $(>1 \mathrm{~mm})$ motion events, which may result in signal changes that cannot be completely modelled. In group studies which comprise the majority of fMRI studies, individual data sets (the so called outliers) ae at worse discarded often without significant repercussions. In individual patient studies such as here, it is imperative to make every attempt at extracting useful information from all datasets. In this work, we evaluate such a scheme whereby individual regressors consisting of a discrete delta function for each scan coinciding with motion events of a magnitude above a pre-set threshold, resulting in a set of so-called scan nulling 
regressors. The purpose of this work was to formally evaluate the efficacy of the scan nulling regressors based on the amount and anatomical extent of the signal variance explained. 


\section{Methods}

34 patients with focal epilepsy who were attending the epilepsy clinics at either the National Hospital for Neurology and Neurosurgery, Queen Square, London, UK, or the National Society for Epilepsy (UK) were studied. These patients were selected if they had frequent IEDs on a recent EEG and form part of a group of 63 patients selected for EEG-fMRI ${ }^{4}$; all patients in whom epileptiform discharges were recorded during fMRI are considered here. The study was approved by the joint ethics committee of the National Hospital for Neurology and Neurosurgery and Institute of Neurology. Subjects gave informed, written consent.

The patients were scanned on a 1.5 Tesla Horizon EchoSpeed MRI scanner (General Electric, Milwaukee, USA) using $\mathrm{T}_{2}{ }^{*}$-weighted single-shot gradient-echo echo-planar images (EPI; TE/TR 40/3000, flip angle: $90^{\circ}, 21 \times 5 \mathrm{~mm}$ interleaved slices, FOV=24 x 24cm, $64 \times 64$ matrix). 700 scans were acquired continuously over a 35-minute period following an initial 12 seconds of scanning to achieve steady state magnetization. For the duration of the functional scans, patients were asked to keep their heads still and to keep their eyes shut. Standard manufacturer-supplied cushions, ear plugs and plastic ear defenders were used.

All patients underwent simultaneous EEG-fMRI acquisitions with 5 patients undergoing two experiments resulting in a total of 39 experiments in 34 patients.

\section{EEG-fMRI experiments}

Using MR-compatible equipment, ten EEG channels were recorded at Fp2/Fp1, F8/F7, T4/T3, T6/T7, O2/O1, Fz (ground) and Pz as the reference (10-20 system), and bipolar electrocardiogram (Krakow, Allen et al. 2000).

All fMRI data were analysed using the SPM2 (Statistical Parametric Mapping) software package (http://www.fil.ion.ucl.ac.uk/spm) and Matlab® (The Mathworks Inc.,USA). Images were slicetime corrected $^{13}$, realigned ${ }^{14}$, and spatially smoothed using an isotropic Gaussian kernel of $8 \mathrm{~mm}$ 
FWHM. Scan realignment proceeded with an iterative estimation of the six rigid body motion parameters.

Offline EEG analysis and fMRI modelling of the IED-related effects was carried out as described previously ${ }^{4}$. Briefly, IED were marked on the EEG and modelled as events via convolution with the canonical haemodynamic response function and its first time derivative as implemented in SPM2.

\section{Motion and fMRI models}

In order to summarise the degree of head motion in each session, we estimated the absolute magnitude of the net displacement vector, $d$, using Pythagoras' theorem based on the translation parameters derived from the scan realignment process. The scan-to-scan displacement was calculated by estimating the absolute magnitude of the first derivative of $\mathrm{d},\left|d^{\prime}\right|$. Individual head jerks were defined by $\left|d^{\prime}\right|>0.2 \mathrm{~mm} / \mathrm{scan}^{4}$.

Effects of motion were modelled in two ways within each design matrix (DM): by the inclusion of a Volterra expansion of the realignment parameters ${ }^{12}$ and by additional 'scan nulling' regressors whereby 4 regressors, each in the form of a Heaviside function corresponding to a scan, are included for each head jerks $(>0.2 \mathrm{~mm})$ spanning a 12 second interval (4 scan repetition times) beginning with the jerk-scan to account for possible T1 effects. Data were high-pass filtered (1/200 seconds cut-off) and pre-whitened to remove slow drifts and correct for temporal non-sphericity ${ }^{15}$. The significance of the effects of interest was assessed using an F-test; maps were thresholded at $P$ $=0.05$, corrected for multiple testing, controlling FWE using Gaussian Random Field Theory. The proportion of brain voxels at which motion effects were significant was estimated for the Volterra and scan nulling regressors. The number of brain voxels was determined automatically as part of the pre-processing (masking) in SPM2. Four dataset-wise summary measures of inter-scan motion were used for comparison with the extent of the motion-related effects: the maximum of $\left|d^{\prime}\right|$ 
$\left(\left|d^{\prime}\right|_{\max }\right),\left|d^{\prime}\right|$ averaged over the dataset $\left(\left|d^{\prime}\right|_{\text {avg }}\right)$, the total number of head jerks and the mean amount of motion per head jerk. 


\section{Results}

Table 1 presents a summary of the degree of motion and anatomical extent of the effect for each case.

The session-wise mean inter-scan displacement averaged over the group was $0.06 \mathrm{~mm}( \pm 0.05$; range: 0.02-0.19; median: 0.05); the mean session-wise maximum inter-scan displacement was $3.38 \mathrm{~mm}( \pm 7.44$; range: $0.17-37.2$; median: 1.12$)$; the mean number of head jerks was $30.7( \pm 46.5$; range: $0-235$; median: 14$)$; the mean number of scan nulling regressors was $85.6( \pm 107$; range: 0 518; median 36). In 4 experiments (\#6, 11, 20 and 23b), there were no head jerks and in four others there were head jerks equivalent to a displacement of $1 \mathrm{~cm}$ or more: \#7a, 14 and 21.

The F-test across all motion-related regressors revealed a significant effect over the majority of brain voxels in $37 / 39$ of cases, including $15 / 16$ cases with a maximum inter-scan displacement below $1 \mathrm{~mm}$. There was a significant effect for the scan nulling regressors over the majority of brain voxels in $14 / 39$ of cases, including 5/16 cases with a maximum inter-scan displacement below $1 \mathrm{~mm}$. As a general rule, motion effects tended to be stronger in cortical than in subcortical brain regions. Taking this into account, scan nulling effects were significant in the majority of the cortex in approximately $50 \%$ of cases. Two representative examples (cases \# 2 and 18) are shown in figures 1 and 2.

The relationship between the proportion of brain voxels (extent) with significant motion-related signal explained by the scan nulling regressors and each of the four summary (experiment-wise) measures of inter-scan motion are plotted in figure 3. We observe a general tendency for the number of scan nulling-affected voxels to increase with the amount of motion for all three measures shown. We note that motion-related effects can be important ( $>40 \%$ of the brain) even for experiments with low $\left(\left|d^{\prime}\right|_{\text {avg }}<0.1 \mathrm{~mm} ;\left|d^{\prime}\right|_{\max }<0.5 \mathrm{~mm}\right)$ degrees of motion. We also note that 
the relationship is not monotonous: Cases \#26 and \#37 stand out because while having large numbers of head jerks, the anatomical extent of the scan nulling effect is relatively small. 


\section{Discussion}

We have evaluated the effectiveness of including two types of model for motion-related effects in the analysis of fMRI data acquired in patients with focal epilepsy: the first is Friston's Volterra expansion of the six realignment parameters to account for spin excitation history effects across successive scans ${ }^{12}$; the second is an ad hoc method that attempts to account for effects due to very large motion events (head jerks) by effectively removing the effected scans from the analysis 4 . Our results demonstrate the general effectiveness of the approach in terms of the proportion of brain voxels for which a significant amount of additional variance is explained by motion-related regressors. Specifically, we have confirmed the Volterra component to be effective even for very small amounts of motion while the addition of scan nulling of the model is efficient for datasets with higher, but commonly observed degrees of motion.

The scan nulling part of the model is mathematically akin to extracting individual volumes from the time series, and is a trade-off between discarding the entire dataset or removing individual (motion-affected) volumes from the dataset on one hand and conventional modelling approaches on the other. Compared to dataset segmentation, this approach has the advantage of preserving temporal continuity and avoids potential errors introduced by scan removal followed by temporal interpolation. Alternative methods that have been proposed to limit the impact of motion on the statistical analysis of fMRI data include data correction methods which rely on modelling of specific artefact generation mechanisms, such as the Volterra expansion used here, and others such as modelling motion-related geometric deformations in EPI ${ }^{11,16,17}$ and spatiotemporal ICA ${ }^{18}$. These methods have been shown to be generally effective but may suffer from modelling limitations in cases of extreme motion and where motion occurs during EPI navigator or data acquisition. More recently a novel method has been proposed which can be likened to the scan nulling approach proposed here in that it attempts to address the issue of motion-related signal changes irrespective of the underlying mechanism ${ }^{19}$. The method is based on the assumption that 
motion has a large impact on the time course of the statistical residuals because they tend to have a relatively large spatial extent. This method assigns motion-dependent weights to each image in the GLM and therefore may be considered conceptually similar to the scan nulling approach used here. The results of a previous analysis of the effects of interest (BOLD changes related to epileptiform discharges) were in line with previous similar studies in terms of yield (proportion of cases with significant activation) while the degree of anatomical concordance of the activation patterns with independently assessed generator localisation (when possible) was high, particularly for positive BOLD changes ${ }^{4}$, consistent with effective false positive control. In this work, we focused on the amount and anatomical distribution of the variance explained by the motion part of the model. We evaluated the effectiveness of the motion-related regressors in experimental data acquired in patients with epilepsy. Other evaluation approaches are possible, such as using data from controlled experiments in which artificial motion is imposed on a phantom or human head. We could also have chosen to compare the epilepsy-related activation patterns obtained for different models (e.g. with and without scan nulling). However this approach is limited by a lack of knowledge of the true brain activity, making it impossible to infer the origin of every activation cluster. Instead, the approach chosen assesses the statistical significance of the additional modelling terms.

We identified two cases which deviated exceptionally from a monotonous trend between the amount of motion (\# of jerks) and the extent of the effect. This may reflect a number of factors, including: the degree to which the measure of motion chosen (linear shift) reflects true motion and the lack of a proper model for large motion events (ad hoc nature of the modelling approach). The use of more sophisticated measures of motion (i.e. including rotation, which of course will be more computationally demanding) may alleviate this problem. Furthermore, although the scan nulling regressors are designed to coincide with large motion events, they can account for any signal changes specific to each scan irrespective of the underlying mechanism. In cases with large 
motion, the patterns observed in this study resembled those typically associated with motion effects.

The choice of a threshold level for the amount of motion that defines an event to be modelled by scan nulling is arbitrary. The value of $0.2 \mathrm{~mm}$ is low enough to be generally considered well within an acceptable degree of motion and as such may be considered a conservative choice; the effects of varying this value on the findings need to be considered.

One of the theoretical weaknesses of the scan nulling approach is its impact on the number of regressors used, which adds to the size of the model as a function of the amount of motion. This can be statistically inefficient, particularly if the scan nulling effect is small. Although one could envisage a modelling strategy whereby two models are tested, namely with and without scan nulling regressors, this can be problematic because of the difficulty of comparing models based on the results in relation to the effects of interest. One could start by implementing a model with scan nulling and test its effectiveness using the approach devised here based on the anatomical extent of the $\operatorname{SPM}\{F\}$, and make a decision about whether to use this model or devise a second model without the scan nulling. This would require the selection of a second (in addition to the jerk detection criterion) arbitrary selection criterion based on the spatial extent and significance of the motion effect. An alternative approach would be to base this choice on the amount of session-wise motion alone. However, the non-monotonous relationship between motion (at least the measures examined here) and motion signal effect makes this difficult. This would also require the choice of a (arbitrary) threshold level for the summary measure of motion. This problem is avoided in the method by Diedrichsen which uses scan-specific weights (between 0 and 1) derived from variance estimates ${ }^{19}$. However, this method is designed to limit the impact of unique, discrete motion events and therefore may not be particularly suited to protect against false positives caused by, stimulus (or event) correlated motion. In contrast, the scan nulling approach is designed to 'steal' power from the effects of interest in these circumstances. 
In this work, we analysed data acquired with the head restraint method usually employed in MR scanners, namely the soft cushions and straps supplied as standard by the scanner manufacturer. More sophisticated head restraint methods are available, such as vacuum cushions ${ }^{20}$. In a different but similar group of patients scanned with a vacuum cushion in our lab, the mean inter-scan displacement with the vacuum cushion was $30 \%$ smaller and the number of jerks $41 \%$ lower than observed here; however, the series-wise maximum inter-scan displacement averaged over the group was $86 \%$ smaller. These results reflect the fact that the vacuum cushion system appears effective in eliminating large head jerks (say, > 1mm) but less effective at reducing the smaller motion events. This suggests that the motion modelling approach is useful even with the use of a vacuum cushion.

We conclude that motion-related effects must be taken into account in the modelling on fMRI data acquired in patients with epilepsy and that the proposed approach can be effective. 


\section{Acknowledgements}

This work was funded by the Wellcome Trust and the Medical Research Council (UK). The support of the National Society for Epilepsy is acknowledged. Thanks to Dr Roman Rodionov for his comments on the manuscript. 
Table

Table 1. Cases, degree of motion and extent of effect

\begin{tabular}{|c|c|c|c|c|c|c|}
\hline \multirow[t]{2}{*}{ Case \# } & \multicolumn{3}{|c|}{ Motion } & \multicolumn{3}{|c|}{ Motion effect } \\
\hline & $\begin{array}{c}\left|d^{\prime}\right| \text { avg } \\
(\mathrm{mm})\end{array}$ & $\begin{array}{c}\left|\mathbf{d}^{\prime}\right| \text { max } \\
(\mathrm{mm})\end{array}$ & $\begin{array}{c}\text { Jerks } \\
(\#)\end{array}$ & $\begin{array}{c}\text { All motion } \\
\text { (\% brain) }\end{array}$ & $\begin{array}{l}\text { Volterra } \\
\text { (\% brain) }\end{array}$ & $\begin{array}{c}\text { Scan nulling } \\
\text { (\% brain) }\end{array}$ \\
\hline 1 & 0.03 & 0.36 & 5 & 50.3 & 53.6 & 0.3 \\
\hline 2 & 0.05 & 0.86 & 20 & 96.4 & 73.1 & 81.5 \\
\hline 3 & 0.03 & 0.49 & 4 & 16.3 & 25.0 & 0.2 \\
\hline 4 & 0.12 & 3.18 & 71 & 85.1 & 87.0 & 99.2 \\
\hline 5 & 0.05 & 1.59 & 34 & 88.5 & 87.0 & 13.1 \\
\hline 6 & 0.02 & 0.19 & 0 & 90.1 & 90.1 & 0.0 \\
\hline $7 a$ & 0.08 & 14.1 & 14 & 99.6 & 80.7 & 16.8 \\
\hline $7 \mathrm{~b}$ & 0.02 & 0.23 & 1 & 65.8 & 62.4 & 6.8 \\
\hline 8 & 0.04 & 0.29 & 3 & 99.4 & 99.2 & 2.1 \\
\hline 9 & 0.03 & 0.38 & 1 & 96.1 & 96.2 & 1.4 \\
\hline 10 & 0.04 & 0.27 & 5 & 81.6 & 99.9 & 0.0 \\
\hline 11 & 0.03 & 0.17 & 0 & 94.7 & 94.7 & 0.0 \\
\hline 12 & 0.03 & 0.39 & 6 & 99.8 & 92.6 & 61.6 \\
\hline 13 & 0.05 & 1.12 & 30 & 86.7 & 45.3 & 25.7 \\
\hline 14 & 0.16 & 26.2 & 66 & 100.0 & 100.0 & 85.7 \\
\hline 15 & 0.05 & 0.61 & 18 & 95.0 & 60.5 & 64.9 \\
\hline 16 & 0.18 & 32.5 & 39 & 100.0 & 98.8 & 88.7 \\
\hline 17 & 0.08 & 1.16 & 45 & 98.5 & 83.5 & 56.5 \\
\hline 18 & 0.04 & 1.89 & 9 & 76.5 & 61.2 & 12.4 \\
\hline 19 & 0.07 & 1.45 & 39 & 98.4 & 60.1 & 91.8 \\
\hline 20 & 0.06 & 0.25 & 0 & 72.0 & 87.0 & 2.4 \\
\hline 21 & 0.11 & 14.1 & 20 & 99.9 & 93.2 & 99.0 \\
\hline 22 & 0.06 & 1.75 & 27 & 74.4 & 69.5 & 7.5 \\
\hline $23 a$ & 0.06 & 3.63 & 5 & 99.4 & 99.0 & 23.5 \\
\hline $23 b$ & 0.03 & 0.17 & 0 & 95.7 & 95.7 & 0.0 \\
\hline $25 a$ & 0.03 & 0.29 & 25 & 99.8 & 71.2 & 87.5 \\
\hline $25 b$ & 0.04 & 2.39 & 2 & 90.2 & 87.6 & 3.9 \\
\hline 26 & 0.19 & 1.45 & 235 & 30.2 & 8.6 & 6.3 \\
\hline $27 a$ & 0.12 & 3.9 & 87 & 100.0 & 84.2 & 99.6 \\
\hline $27 b$ & 0.14 & 2.44 & 118 & 99.7 & 91.3 & 99.4 \\
\hline 29 & 0.05 & 0.55 & 37 & 96.2 & 85.1 & 65.3 \\
\hline 30 & 0.03 & 0.43 & 8 & 82.2 & 85.6 & 3.2 \\
\hline 31 & 0.03 & 1.57 & 14 & 99.5 & 93.8 & 87.1 \\
\hline 35 & 0.05 & 0.5 & 5 & 95.1 & 95.6 & 7.8 \\
\hline 36 & 0.02 & 1.42 & 7 & 99.4 & 99.4 & 42.3 \\
\hline 37 & 0.13 & 2.32 & 135 & 43.5 & 58.9 & 1.5 \\
\hline $38 a$ & 0.05 & 1.64 & 8 & 96.1 & 95.9 & 7.8 \\
\hline $38 b$ & 0.06 & 0.57 & 30 & 99.4 & 95.5 & 19.9 \\
\hline 39 & 0.02 & 0.51 & 4 & 84.5 & 85.2 & 8.8 \\
\hline
\end{tabular}




\section{Figure captions}

Figure 1. Extent of motion effects: Case \#2. The degree of motion in this case was close to the median across the group: $\left|d^{\prime}\right|_{\text {avg }}=0.05 \mathrm{~mm} ;\left|d^{\prime}\right|_{\max }=0.86 \mathrm{~mm} ; 20$ head jerks. SPM\{F\}'s: Top: all motion regressors; middle: Volterra regressors; bottom: scan nulling regressors. The design matrix and contrast are represented in the left, with the supra-threshold voxels overlaid on cross-sections of the mean image on the right. The cross-hair is at the global maximum. The proportion of the brain with a significant overall motion effect was $96.4 \%$, with $96.4 \%$ for the scan nulling component and $73.1 \%$ for the Volterra component.

Figure 2. Extent of motion effects: Case \#18. The degree of motion in this case was close to the median across the group: $\left|d^{\prime}\right|_{\text {avg }}=0.04 \mathrm{~mm} ;\left|d^{\prime}\right|_{\max }=1.89 \mathrm{~mm} ; 9$ head jerks. SPM\{F\}'s: Top: all motion regressors; middle: Volterra regressors; bottom: scan nulling regressors. The design matrix and contrast are represented in the left, with the supra-threshold voxels overlaid on the cross sections of the mean image on the right. The cross-hair is at global maximum. The proportion of the brain with a significant overall motion effect was $76.5 \%$, with $12.4 \%$ for scan nulling component and $61.2 \%$ for the Volterra component.

Figure 3. Relationship between degree of motion and anatomical extent of motion effects. Percentage of the brain over which there is a significant effect of motion modelled by the scan nulling regressors. The degree of motion is in mm for $\left|\mathrm{d}^{\prime}\right|_{\text {avg }},\left|\mathrm{d}^{\prime}\right|_{\max }$, Mean jerk motion (the interscan displacement averaged over jerk events) and number of jerks. 

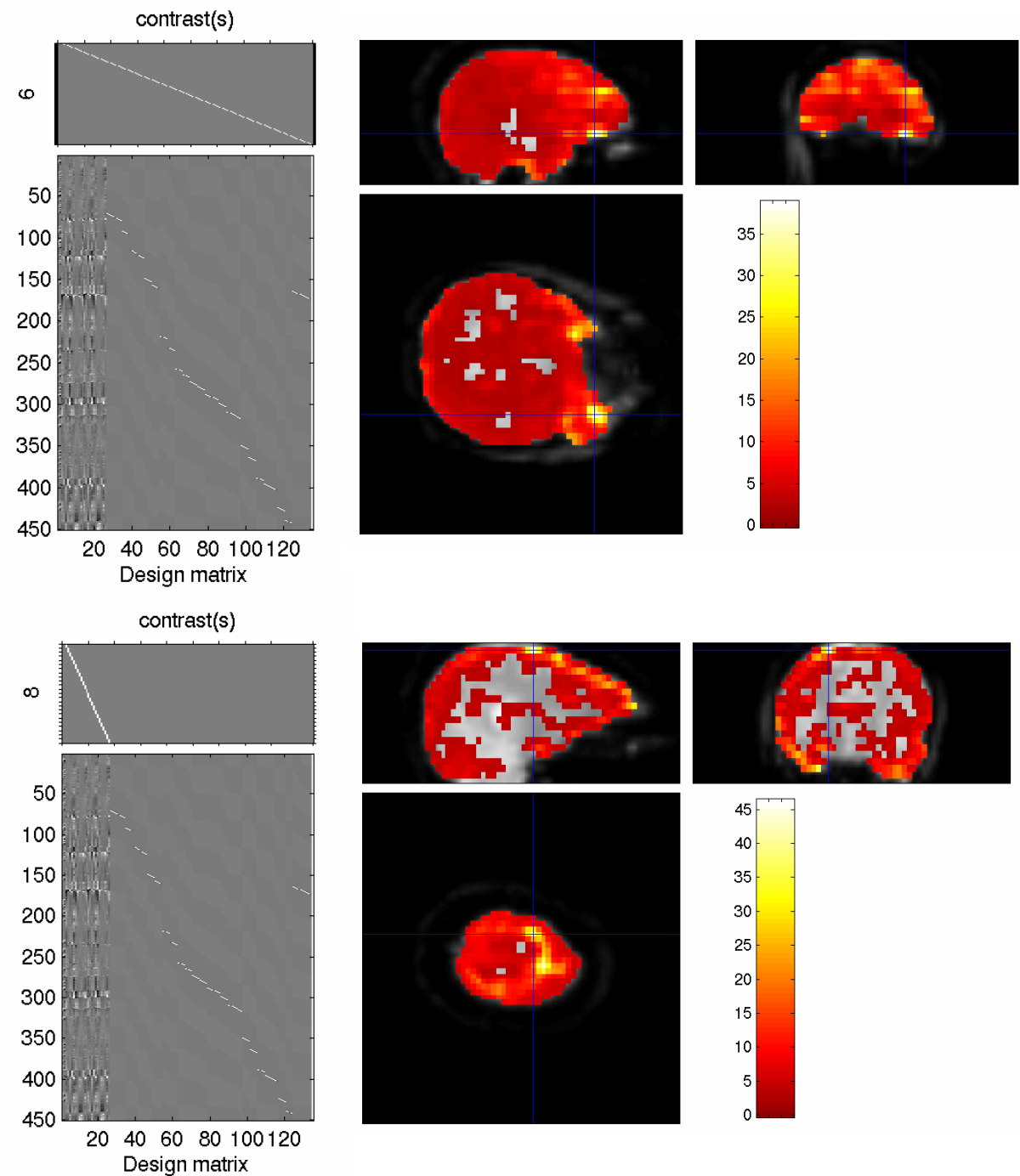

Design matrix
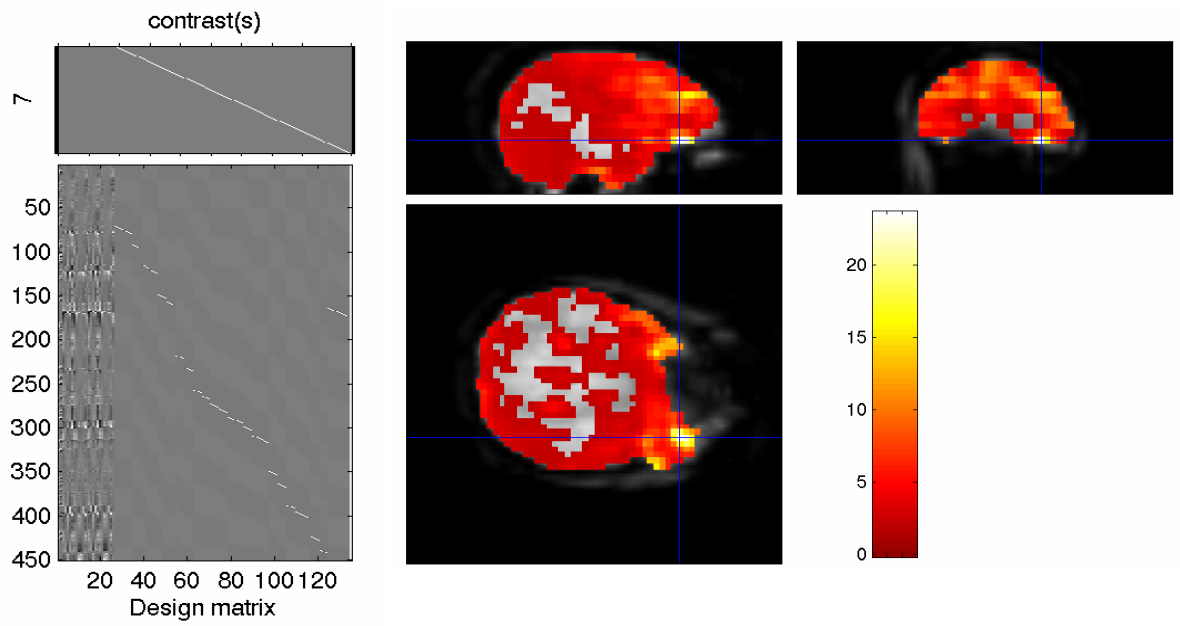

Figure 1 

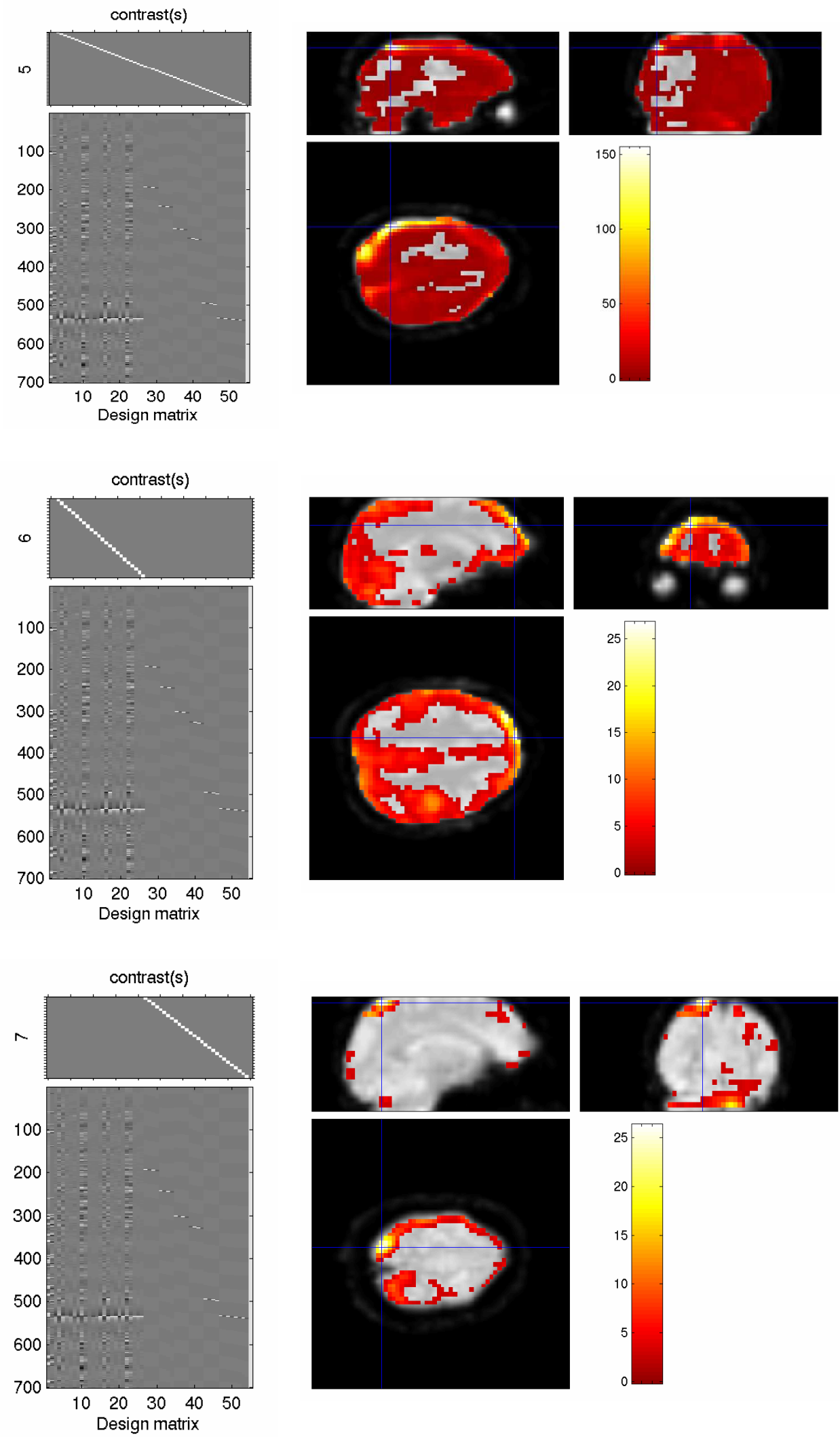

Figure 2 


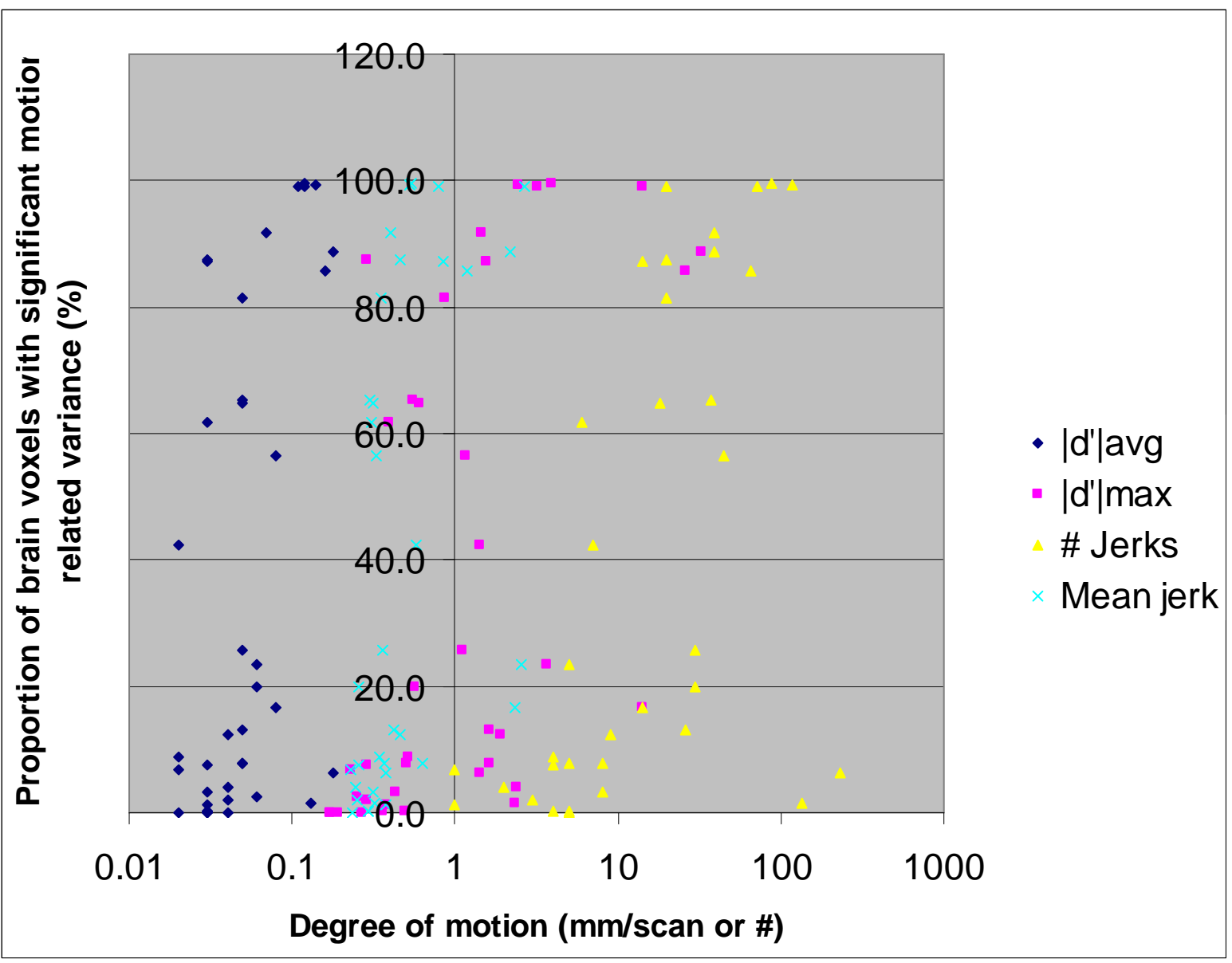

Figure 3. 


\section{References}

1. Friston KJ, Holmes AP, Worsley KJ, Poline JP, Frith CD, Frackowiak RSJ. Statistical Parametric Maps in Functional Imaging: A General Linear Approach. Hum-Brain-Mapp 1995;2:189-210.

2. Krakow K, Lemieux L, Messina D, Scott CA, Symms MR, Duncan JS, Fish DR. Spatiotemporal imaging of focal interictal epileptiform activity using EEG-triggered functional MRI. Epileptic Disord 2001 Jun;3(2):67-74.

3. Krakow K, Woermann FG, Symms MR, Allen PJ, Lemieux L, Barker GJ, Duncan JS, Fish DR. EEG-triggered functional MRI of interictal epileptiform activity in patients with partial seizures. Brain 1999 Sep;122 ( Pt 9):1679-88.

4. Salek-Haddadi A, Diehl B, Hamandi K, Merschhemke M, Liston A, Friston K, Duncan JS, Fish DR, Lemieux L. Hemodynamic correlates of epileptiform discharges: an EEG-fMRI study of 63 patients with focal epilepsy. Brain Res 2006 May;1088(1):148-66.

5. Diehl B, Salek-Haddadi A, Fish DR, Lemieux L. Mapping of spikes, slow waves, and motor tasks in a patient with malformation of cortical development using simultaneous EEG and fMRI. Magn Reson Imaging 2003 Dec;21(10):1167-73.

6. Kobayashi E, Bagshaw AP, Benar CG, Aghakhani Y, Andermann F, Dubeau F, Gotman J. Temporal and extratemporal BOLD responses to temporal lobe interictal spikes. Epilepsia 2006 Feb;47(2):343-54.

7. Al Asmi A, Benar CG, Gross DW, Khani YA, Andermann F, Pike B, Dubeau F, Gotman J. fMRI Activation in Continuous and Spike-triggered EEG-fMRI Studies of Epileptic Spikes. Epilepsia 2003 Oct;44(10):1328-39.

8. Hajnal JV, Myers R, Oatridge A, Schwieso JE, Young IR, Bydder GM. Artifacts due to stimulus correlated motion in functional imaging of the brain. Magn-Reson-Med 1994;31:283-91.

9. Lund TE, Norgaard MD, Rostrup E, Rowe JB, Paulson OB. Motion or activity: their role in intra- and inter-subject variation in fMRI. NeuroImage 2005 Jul;26(3):960-4.

10. Friston KJ, Ashburner J, Frith CD, Poline JB, Heather JD, Frackowiak RSJ. Spatial registration and normalisation of images. Hum-Brain-Mapp 1995;2:165-89.

11. Andersson JLR, Hutton C, Ashburner J, Turner R, Friston KJ. Modeling geometric deformations in EPI time series. NeuroImage 2001;13:903-19.

12. Friston KJ, Williams S, Howard R, Frackowiack RSJ, Turner R. Movement-related effects in fMRI time-series. Magn-Reson-Med 1996;35:346-55.

13. Henson RNA, Buchel C, Josephs O, Friston KJ. The slice-timing problem in event-related fMRI. Neuroimage 1999;9:125. 
14. Friston KJ, Ashburner J, Poline JB, Frith CD, Heather JD, Frackowiak RS. Spatial Registration and Normalization of Images. Hum Brain Mapp 1995;2:165-89.

15. Friston KJ, Josephs O, Zarahn E, Holmes AP, Rouquette S, Poline J. To smooth or not to smooth? Bias and efficiency in fMRI time-series analysis. Neuroimage 2000 Aug;12(2):196208.

16. Hutton C, Bork A, Josephs O, Deichmann R, Ashburner J, Turner R. Image distortion correction in fMRI: a quantitative evaluation. NeuroImage 2002;16:217-40.

17. Grootoonk S, Hutton C, Ashburner J, Howseman AM, Josephs O, Rees G, Friston KJ, Turner R. Characterization and correction of interpolation effects in the realignment of fMRI time series. NeuroImage 2000 Jan;11(1):49-57.

18. De Martino F, Gentile F, Esposito F, Balsi M, Di Salle F, Goebel R, Formisano E. Classification of fMRI independent components using IC-fingerprints and support vector machine classifiers. NeuroImage 2006 Oct.

19. Diedrichsen J, Shadmehr R. Detecting and adjusting for artifacts in fMRI time series data. NeuroImage 2005 Sep;27(3):624-34.

20. Benar C, Aghakhani Y, Wang Y, Izenberg A, Al Asmi A, Dubeau F, Gotman J. Quality of EEG in simultaneous EEG-fMRI for epilepsy. Clin Neurophysiol 2003 Mar;114(3):569-80. 